\title{
Probabilistic Modelling for Frequency Response Functions and Transmissibility Functions with Complex Ratio Statistics
}

\author{
Meng-Yun Zhao \\ Department of Civil Engineering, Hefei University of Technology, China.E-mail: zmyhfut@163.com
}

Wang-Ji Yan*

Institute for Aerospace Technology \& The Composites Group, The University of Nottingham, United Kingdom. Department of Civil Engineering, Hefei University of Technology,China. E-mail: civilyanwj@gmail.com

Wei-Xin Ren

Department of Civil Engineering, Hefei University of Technology,China.E-mail: renwx@hfut.edu.cn

Michael Beer

Institute for Risk and Reliability, Leibniz Universität Hannover, Germany. E-mail: beer@irz.uni-hannover.de

The distributions of ratios of random variables arise in many applied problems such as in structural dynamics working with frequency response functions (FRFs) and transmissibility functions (TFs). When analysing the distribution properties of ratio random variables through the definition of probability density functions (PDF), the problem is usually accompanied by multiple integrals. In this study, a unified solution is presented to efficiently calculate the PDF of a ratio random variable with its denominator and numerator specified by arbitrary distributions. With the use of probability density transformation principle, a unified expression can be derived for the ratio random variable by reducing the concerned problem into two-dimensional integrals. As a result, the PDFs of the ratio random variable can be efficiently computed by using effective numerical integration techniques. Finally, based on the vibration tests performed on the Alamosa Canyon Bridge, the proposed method is applied to the data to quantify the uncertainty of FRFs and TFs.

Keywords: Frequency response function, transmissibility function, probability density function, ratio distribution, numerical integration, structural dynamics

\section{Introduction}

Frequency response functions (FRF) and transmissibility functions $(\mathrm{TF})$ are viewed as the most fundamental functions in structural dynamics (Yan and Ren, 2016a; 2016b; 2018a). They have been widely regarded to be powerful candidates for structural health monitoring (Maia et al., 2011; Mao, 2012). Due to the inherent randomness of measurements and variability of environmental conditions, uncertainty impacts their applications significantly. FRFs and TFs are complex-valued ratio random variables composed of both real and imaginary parts. Therefore, it is important to explore new approaches for quantifying uncertainty of complex ratio random variables.

Currently, ratio distributions have been studied by a large panel of researchers in many fields like biological and physical sciences and econometrics (Hinkley, 1969; Korhonen and Narula, 1989). However, most of the research on ratio random variables is restricted by assuming that the denominator and numerator are independent and follow the same distribution. More importantly, the available theories are not applicable to treating complex-valued ratio random variables. To address this issue, new theorems have been obtained on multivariate circularly symmetric complex Gaussian ratio distribution as well as some useful properties deduced as corollaries and lemmas are proved mathematically (Yan and Ren, 2016a;2016b). Unfortunately, the new distribution is limited to tackling the ratio random variables with denominator and numerator following complexvalued Gaussian distribution with zero mean.

In practical applications it has been proven that the FFT coefficients may deviate from a Gaussian distribution, and the circularly symmetric complex normal ratio distribution may cause unexpected errors during the process of uncertainty quantification (Yan and Ren, 2018b). Therefore, one needs to propose a more versatile

Proceedings of the 29th European Safety and Reliability Conference.

Edited by Michael Beer and Enrico Zio

Copyright (c) 2019 European Safety and Reliability Association.

Published by Research Publishing, Singapore. 
way to compute the ratio distribution following arbitrary probability distributions.

To address the issue, this study aims at proposing a unified approach which can efficiently calculate the PDF of complex ratio random variables with its denominator and numerator specified by arbitrary probability distributions by reducing the concerned problem into two-dimensional integrals. The formula can be evaluated by using effective numerical integration techniques. According to the characteristics of the unified integral formula obtained, 2-dimensional Gaussian-Hermite based on sparse-grid quadrature rule is employed to calculate the solution efficiently. Using the vibration tests performed on the Alamosa Canyon Bridge, the uncertainties of FRFs and TFs are quantified based on the proposed method.

\section{Definition of FRF and TF}

The FRF denoted by $H_{i, j}\left(\omega_{k}\right)$ between the output of the $i$-th dof $\left(X_{i}\left(\omega_{k}\right)\right)$ and the input applied at the $j$-th $\operatorname{dof}\left(F_{j}\left(\omega_{k}\right)\right)$ is defined as (Yan and Ren, 2018):

$$
H_{i, j}\left(\omega_{k}\right)=\frac{X_{i}\left(\omega_{k}\right)}{F_{j}\left(\omega_{k}\right)}
$$

A TF $T_{i, o}\left(\omega_{k}\right)$ is defined as the ratio of FFT coefficient of an arbitrary response $X_{i}\left(\omega_{k}\right)$ to a reference response $X_{o}\left(\omega_{k}\right)$, which is a complexvalued random variable composed of both real and imaginary parts which are correlated with each other (Yan and Ren, 2016a,b):

$$
T_{i, o}\left(\omega_{k}\right)=\frac{X_{i}\left(\omega_{k}\right)}{X_{o}\left(\omega_{k}\right)}
$$

where all ' $\omega_{k}$ ' shown in the bracket denote frequency.

As is seen from Eq.(1) and (2), it is not difficult for one to figure out that FRF and TF are complex-valued ratio random variables composed of both real and imaginary parts which are correlated with each other. Based on the unique mathematical structure of the two classical functions, a unified approach will be proposed in the following sections to compute the PDF of complex ratio random variables.

\section{PDF of Complex Ratio Random Variable}

To avoid direct using of the definition to calculate multiple CDF and obtaining complex ratio distribution function using partial derivatives, we introduce the principle of probabilistic transformation of random vectors. As a result, complex-valued ratio random variable following arbitrary distribution can reduce to a problem with a double dimension integral.

For the convenience of illustration, the FFT coefficients are denoted by $\mathbf{Y}=\left\{Y_{1}, Y_{0}\right\}^{T}$. Assume that FRF and TF at any frequency point are mathematically denoted as $U=Y_{1} / Y_{0}$, which is defined as the ratio of FFT coefficient of $Y_{1}$ to $Y_{0}$. The frequency point is omitted here. Direct application of principle of probabilistic transformation of random vectors is impossible in that the order of $\mathbf{Y}$ and $U$ are not equal. To address this difficulty, a new random vector $\mathbf{Q}$ with its PDF denoted by $p_{\mathbf{Q}}(\boldsymbol{q})$ is constructed as $\mathbf{Q}=$ $\left\{Y_{0}, U\right\}^{T}$ (Yan and Ren, 2016a).

In such a way, one can relate random vectors $\mathbf{Q}$ and $\mathbf{Y}$ through a linear transformation $\mathbf{Q}=\mathbf{W} \mathbf{Y}$. According to the principle of probabilistic transformation of random vectors (Yan and Ren, 2016a)

$$
p_{\boldsymbol{Q}}(\boldsymbol{q})=p_{\boldsymbol{Y}}(\boldsymbol{y}) /\left|J_{W}\right|
$$

Here $J_{W}=\left[\begin{array}{cc}\mathbf{w}^{\Re} & -\mathbf{w}^{\Im} \\ \mathbf{w}^{\Im} & \mathbf{w}^{\Re}\end{array}\right]$ denotes Jacbian matrix. It is worth noting that $\left|\operatorname{det} J_{W}\right|$ can be rearranged as

$$
\left|\operatorname{det} J_{W}\right|=\left|y_{0}\right|^{-2}
$$

It is noted that there is a functional relationship $\mathbf{Y}=Y_{0} \widetilde{\mathbf{U}}=Y_{0}\{\mathbf{1} \mathbf{U}\}$ between $\mathbf{Y}$ and $U$. And considering Eq.(3) and (4), $p_{\mathbf{Q}}(\boldsymbol{q})$ can be expressed in terms of random variable $Y_{0}$ and random vector $U$ as

$$
p_{\boldsymbol{Q}}(\boldsymbol{q})=\left|y_{0}\right|^{2} p_{\boldsymbol{Y}}\left(y_{0} \widetilde{\boldsymbol{u}}\right)
$$

As a result, the PDF of $U$ is given by

$$
p_{\boldsymbol{U}}(\boldsymbol{u})=\int_{-\infty}^{+\infty} \int_{-\infty}^{+\infty}\left|y_{0}\right|^{2} p_{\boldsymbol{Y}}\left(y_{0} \widetilde{\boldsymbol{u}}\right) d y_{0}^{\Re} d y_{0}^{\Im}
$$

\section{Numerical Solution}

As is seen from Eq.(6), whether closed-form analytical solution is available or not depends on the formula $p_{\mathbf{Y}}\left(y_{0} \widetilde{\mathbf{u}}\right)$. Obviously, it is highly non- 
trivial to obtain the closed-form analytical solution for arbitrary ratio random variables. One natural way to address the problem mentioned in the above is to resort to efficient numerical algorithms. It is not difficult to figure out that computing the PDF for a complex-valued ratio random variable involves calculating the 2dimensional Gaussian-Hermite. The GaussHermite quadrature formula for a 2-dimensional integral is given by

$$
\begin{aligned}
& \int_{-\infty}^{+\infty} \int_{-\infty}^{+\infty} f\left(x_{1}, x_{2}\right) e^{-\left(x_{1}^{2}+x_{2}^{2}\right)} d x_{1} d x_{2} \\
& \quad \approx \sum_{i_{1}=1}^{m_{1}} \sum_{i_{2}=1}^{m_{2}} \omega_{x_{i_{1}}} \omega_{x_{i_{2}}} f\left(x_{i_{1}}, x_{i_{2}}\right)
\end{aligned}
$$

As is seen from Eq.(7), the total number of points to be calculated increases exponentially with the dimension. Therefore, the computational burden also increases significantly with the dimension. To address the drawbacks, a fast quadrature approach based on the sparse-grid theory will be employed to address the curse of dimensionality problem. The locations and weights of the univariate quadrature points with a range of accuracy levels are determined by asymptotic approximations method. Then the univariate quadrature point sets are extended to form a multi-dimensional grid using the sparsegrid theory. The sparse-grid method is based on the Smolyak rule, given by Heiss and Winschel (2008). One can refer to Bin and Ming (Bin et al., 2012) for more details about the algorithm.

\section{Case Study}

To further illustrate the accuracy of the proposed method, this section investigates the full-scale field measurement data of the Alamosa Canyon Bridge (Farrar et al., 2000) shown in Fig. 1. Field measurements of the acceleration time histories and the ambient conditions including temperature were provided in the Ref. (Farrar, et al. 2000) as well as the website: http://ext.lanl.gov/projects/damage_id/.

The response measurements are segmented into 330 non-overlapping sequences with each one lasting $16 \mathrm{~s}$. The FFT coefficients can be calculated accordingly for each sequence. As a result, a thorough validation can be implemented in a similar way to MCS with each segment viewed as a random realization. The FRF $\left(H_{4,3}\left(\omega_{k}\right)\right)$ corresponding to the fourth output $X_{4}\left(\omega_{k}\right)$ and the input at the third DOF $F_{3}\left(\omega_{k}\right)$, as well as the TF $\left(T_{20,1}\left(\omega_{k}\right)\right)$ observed in detail by taking the ratio of $X_{20}\left(\omega_{k}\right)$ to $X_{1}\left(\omega_{k}\right)$ are considered as examples. $X_{4}^{(k)}$ is the FFT coefficient of response at the fourth position, while $X_{20}\left(\omega_{k}\right), X_{1}\left(\omega_{k}\right)$ are defined in the same way. $F_{3}\left(\omega_{k}\right)$ is the FFT coefficient of input at the third position. In Fig. 2 and Fig. 4, the red solid line and the green dotted line denotes the $t$ location scale and normal distribution functions of the real part and imaginary part of $X_{4}\left(\omega_{k}\right)$ and $X_{20}\left(\omega_{k}\right)$, while the histograms denote the probability mass functions from 330 segments. As is seen from these figures, one can find that $t$ distribution can fit the histograms better than Gaussian distribution. Therefore, the FRF and TF at some frequency points will employ complex $t$ ratio probabilistic models.

The theoretical values of the marginal PDFs of the real part and imaginary part of $H_{4,3}\left(\omega_{k}\right)$ at $0.139 \pi \mathrm{rad} / \mathrm{s}$ and $T_{20,1}\left(\omega_{k}\right)$ at $3.183 \pi \mathrm{rad} / \mathrm{s}$ are computed using Eq. (6) with the help of Eq.(7), whose results are shown in Fig. 3 and 5 by comparing to the histograms. It can be seen from Figs. 3 and 5 that the solid curves agree well with the histograms, implying that the FRF and TF are well approximated by the complicated $t$ ratio distribution which are calculated using numerical integration method. Therefore, the unified computational model using efficient numerical method is expected to be efficient to accommodate the uncertainties of the FRF and TF for structures.

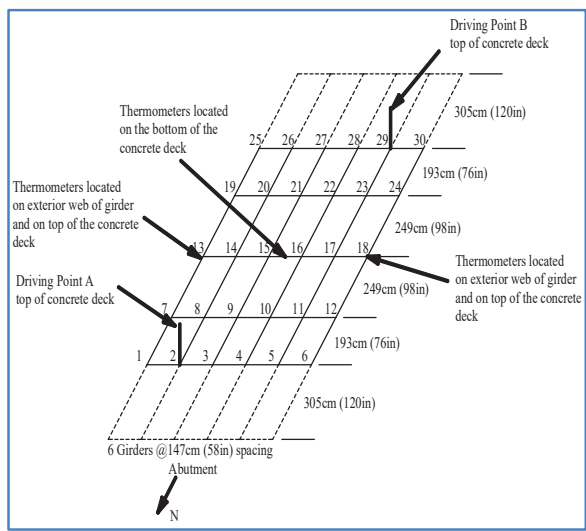

Fig. 1. Positions of the accelerometer of the Alamosa Canyon Bridge (from Farrar, et al. 2000) 


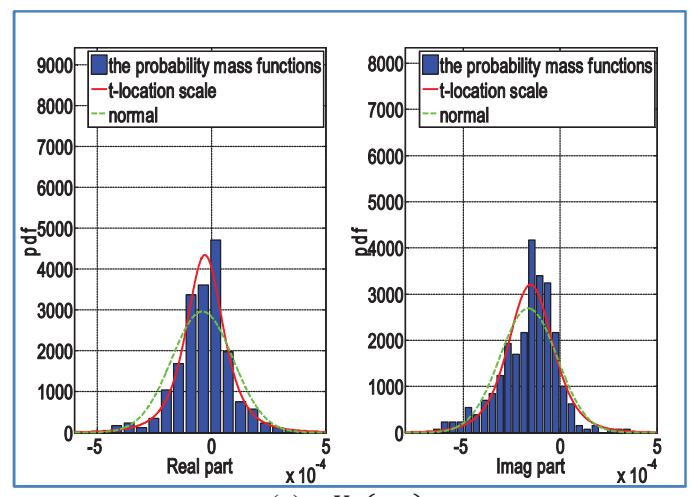

(a) $X_{4}\left(\omega_{k}\right)$

Fig. 2. The theoretical marginal PDFs and the histogram PDFs of the real part and imaginary part of $X_{4}\left(\omega_{k}\right)$ at $0.139 \pi$

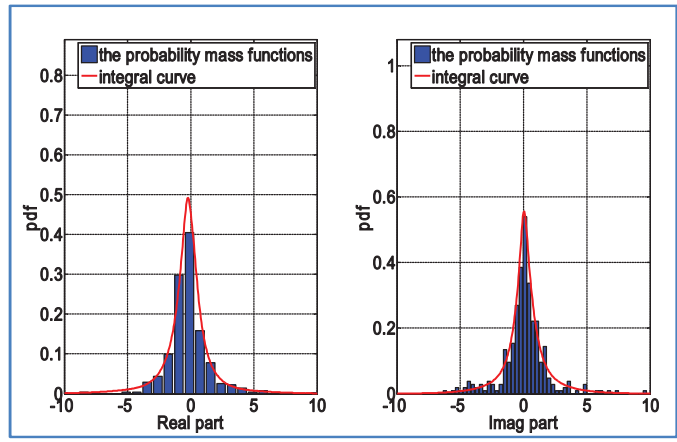

Fig. 3. The theoretical marginal PDFs and the histogram PDFs of the real part and imaginary part of $H_{4,3}\left(\omega_{k}\right)$

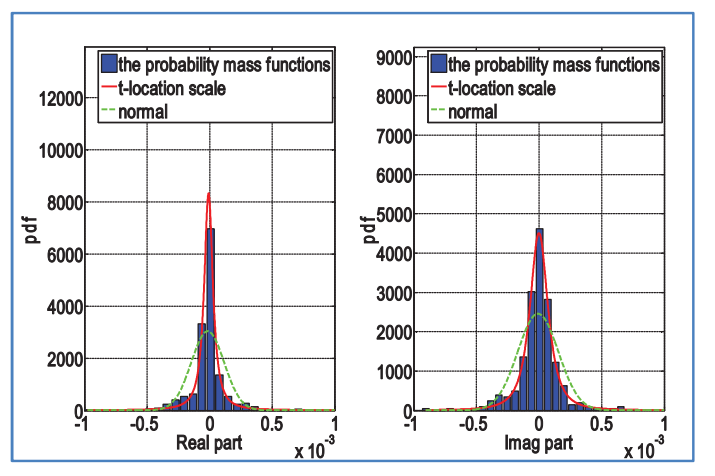

Fig. 4. The theoretical marginal PDFs and the histogram PDFs of the real part and imaginary part of $X_{20}\left(\omega_{k}\right)$ at $3.183 \pi$

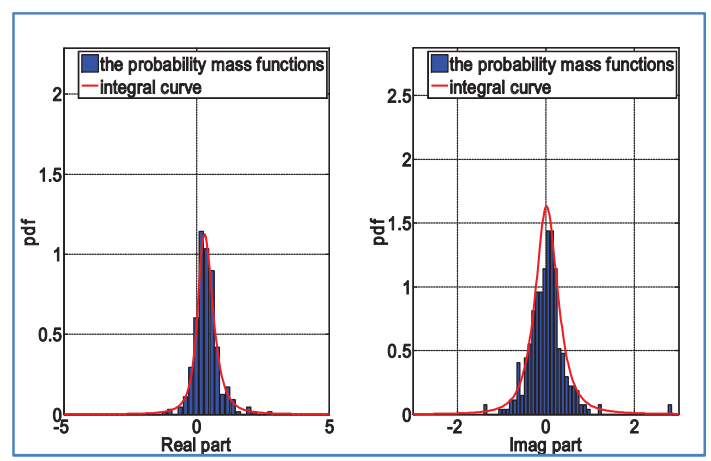

Fig. 5. The theoretical marginal PDFs and the histogram PDFs of the real part and imaginary part of $T_{20,1}\left(\omega_{k}\right)$

\section{Conclusion}

FRF and TF are important tools in various applications including modal analysis and damage detection. PDF plays a vital role in uncertainty quantification for them. On the basis of this kind of functions defined as complex ratio random variables, this paper deduces a unified approach for computing probability distribution of arbitrary complex ratio random variables. The general solution is obtained by efficient numerical integral method, which is based on the sparse-grid theory. Finally, the theory is verified by quantifying the uncertainty of the FRF and TF using the field test data of the Alamosa Canyon Bridge. Results indicates that the unified computational probability model using efficient numerical algorithm proposed in this study can quantify ratio random variables following arbitrary distribution.

\section{Acknowledgement}

Dr. W.J. Yan was supported by the Marie SklodowskaCurie individual Fellowships of the EU under Contract 741284 when visiting the University of Nottingham. The authors would thank Los Alamos National Laboratory for providing the data from the various vibration tests performed on the Alamosa Canyon Bridge to the public.

\section{References}

Bin, J., X. Ming and C. Yang (2012). Sparse-grid quadrature nonlinear filtering. Automatica 48, 327-341. Farrar, C.R., P.J. Cornwell, S.W. Doebling, and M.B. Prime (2000). Structure health monitoring studies of 
the Alamosa Canyon and I-40 Bridges. Office of Scientific \& Technical Information Technical Reports, 2000.

Heiss, F., and V. Winschel (2008). Likelihood approximation by numerical integration on sparse grids. Journal of Econometrics 144, 62-80.

Hinkley D.V. (1969). On the ratio of two correlated normal random variables. Biometrika 56, 635-639.

Korhonen, P.J., and S.C. Narula (1989). The probability distribution of the ratio of the absolute values of two normal variables. Journal of statistical computation and simulation 33, 173-182

Maia, N.M.M., R. Almeida, A.P.V. Urgueira, and R.P.C. Sampaio (2011). Damage detection and quantification using transmissibility. Mechanical Systems and Signal Processing 25, 2475-2483.

Mao, Z. (2012). Uncertainty quantification in vibrationbased structural health monitoring for enhanced decision-making capability, University of California.

Yan, W.J., and W.X. Ren (2016a). Circularlysymmetric complex normal ratio distribution for scalar transmissibility functions. Part I: Fundamentals. Mechanical Systems and Signal Processing 80, 58-77. Yan, W.J., and W.X. Ren (2016b). Circularlysymmetric complex normal ratio distribution for scalar transmissibility functions. Part II: Probabilistic models and validation. Mechanical Systems and Signal Processing 80, 78-98.

Yan, W.J., and W.X. Ren (2018a). Circularlysymmetric complex normal ratio distribution for scalar transmissibility functions. Part III: Application to statistical modal analysis. Mechanical Systems and Signal Processing 98, 1000-1019.

Yan, W.J., and W.X. Ren (2018b). Generalized proper complex Gaussian ratio distribution and its application to statistical inference for frequency response functions. Journal of Engineering Mechanics 144 